\title{
The Effects of Cervical Mobilization Combined with Thoracic Mobilization on Forward Head Posture of Neck Pain Patients
}

\author{
Jaehong Lee, PhD, $\mathrm{PT}^{1)}$, Younghwa Lee, PhD, $\mathrm{PT}^{2}$, \\ Hansoo Kim, PhD, PT ${ }^{3)}$, Jongho LeE, $\mathrm{PhD}^{4}$, \\ 1) Department of Physical Therapy, Daegu Health College \\ 2) Center of Physical Therapy, Kimjun's Orthopaedic Clinic: 77-1 Seomoonro 1 street, Jung-gu, Daegu, \\ 700-251 Republic of Korea. TEL: +82 53-255-8336, FAX: +82 53-255-8336, E-mail: ptspecialist@ \\ naver.com \\ 3) Department of Occupational Therapy, Daegu Health College \\ 4) Department of Sport Management, Namseoul University
}

\begin{abstract}
Purpose] The purpose of the present study was to examine the effects of cervical mobilization combined with thoracic mobilization on neck pain patients' recovery from forward head posture (FHP). [Subjects] Thirty neck pain patients with FHP were randomly assigned to an experimental group $(n=15)$ and a control group $(\mathrm{n}=15)$. [Methods] The experimental group received cervical mobilization combined with thoracic mobilization and the control group received only cervical mobilization. Cranial vertical angle (CVA) and cranial rotation angle (CRA) were measured before and after the experiment. [Results] The experimental group had significantly greater improvements in CVA and CRA than the control group. [Conclusion] Therefore, we recommend cervical mobilization combined with upper thoracic mobilization for the improvement of FHP in neck pain patients.

Key words: Cranial vertical angle, Cranial rotation angle, Forward head posture
\end{abstract}

(This article was submitted Aug. 1, 2012, and was accepted Aug. 30, 2012)

\section{INTRODUCTION}

As people in modern society spend an increasing amount of time sitting in front of computers at work, forward head posture (FHP) has become quite prevalent in recent years. This deformation causes injuries to soft tissues, thereby inducing non-specific neck pain, restricting the range of motion of the joints, and even causing radiating pain or myasthenia, depending on the region of neuromuscular lesions, when the deformation becomes severe ${ }^{1,2)}$. Therefore, recovery from FHP is considered very important for relieving neck pain.

Among treatments aiming to improve the reduced cervical range of motion that results from FHP, spinal mobilizations such as Mulligan mobilization and Gong's mobilization ${ }^{3,4)}$, McKenzie exercise ${ }^{5)}$, and stretching ${ }^{6}$ are known to be effective. Recently, joint mobilization has proven to be effective, and it is now frequently used in clinics ${ }^{7}$.

The joint mobilization used to treat FHP is applied to the cervical spine in most cases. However, since FHP typically shows increased flexion of the lower cervical spine and the upper thoracic spine as well as increased extension of the atlas, the occiput, and the upper cervical spine ${ }^{8)}$, not only the cervical spine, but also the upper thoracic vertebrae require treatment. In a recent study, in which thoracic mobilization was performed on 37 chronic neck pain patients, it was reported that the range of flexion and extension of the cervical spine significantly increased ${ }^{9)}$. Therefore, along with cervical mobilization to restore the cervical spine's normal lordotic angles, the application of thoracic mobilization is also important for cervical spine recovery in the treatment of FHP. However, no study has yet been conducted of the combined application of cervical and thoracic mobilization.

Therefore, the purpose of the present study was to examine the effects of cervical mobilization combined with thoracic mobilization on neck pain patients' recovery from FHP.

\section{SUBJECTS AND METHODS}

The present study was conducted using 30 patients diagnosed with neck pain as subjects. All patients had FHP with $15 \mathrm{~mm}$ or larger anterior weight bearing $(\mathrm{AWB})^{10)}$ in lateral views in plain radiography. Patients were seen at D Hospital in Korea between April and June 2012. They were randomly assigned to an experimental group of 15 patients or a control group of 15 patients. Differences in sex, age, height, and weight between the experimental and control groups were not statistically significant ( $\mathrm{p}>0.05)$; thus, the two groups were considered to be homogeneous.

Selection criteria for the subjects were: those with neck pain, FHP, and $15 \mathrm{~mm}$ or larger AWB. Exclusion criteria were: patients with a history of cervical spine operation, fractures in the cervical spine, rheumatoid arthritis, or 
cervical disc herniation, and those with other contraindications against joint mobilization. The purpose of the present study and general contents of the experiment were sufficiently explained to all the subjects, and written agreements to participation in the study were received from all of the subjects.

In the present study, the experimental group received cervical mobilization combined with thoracic mobilization, and the control group received cervical mobilization only. Both the experimental group and the control group received joint mobilization for 15 minutes each, three times a week, for four weeks. Joint mobilization was applied by a therapist, a certified orthopedic manual therapist with 16 years of experience.

For the cervical mobilization that was performed for both the experimental group and the control group, each patient was instructed to sit on the edge of a hospital bed with his/ her feet touching the floor. The therapist stood on one side of the patient and guided the patient's neck to a passive neutral posture. Then, the therapist adjusted his posture so that one side of the patient's face a into contact with his chest. The therapist then wrapped one arm around the other side of the patient's face and wrapped that hand around the back of the patient's neck while touching the region above the upper bone of the segment to be mobilized with the same hand. The therapist fixed the spinous process of the lower bone of the segment to be mobilized using the thumb and the index finger of the other hand. Then, the therapist performed joint mobilization by gently pushing the fixing hand anteriorly and upward, and pushing the hand wrapping around the patient's face backward to induce cervical retraction. At the end range of the retraction, the therapist created passive extension while increasing the speed using gravity and weight.

For the experimental group, upper thoracic mobilization was performed after cervical mobilization. First, each patient was instructed to sit on the edge of a hospital bed with his/her feet touching the floor and with his hands clasped behind the neck. The therapist stood on one side of the patient and fixed the patient's lumbosacral region using one lower extremity while wrapping his/her hand around the patient's elbow joint, with his hand anterior to the patient, to fix the patient's cervical spine above the segment to be mobilized so that one movement would occur in the region. Then, while fixing the segment below the segment to be mobilized, using the thumb and index finger of his hand behind the patient, the therapist lifted the patient's elbow joint upward and anteriorly to extend the patient's thoracic spine. Next, the therapist pushed the fixing hand forward to apply counter-pressure. The therapist pushed hard at the end range to increase mobility.

To examine changes in FHP in the experimental and control groups, cranial vertical angle (CVA) and cranial rotation angle (CRA) were measured before the treatment and after the treatment which lasted for four weeks (Fig.1).

Before measuring the CVA and CRA, self-balance posture (SBP) was used to initiate the natural head posture (NHP). The SBP was achieved by having the subject perform the maximum level of flexion or extension of his/her cervical spine and gradually reduce the degree of flexion or extension

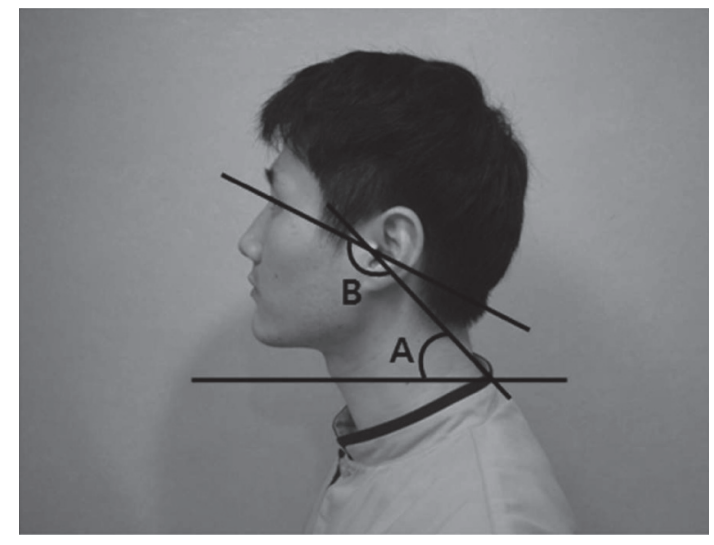

Fig. 1. A: cranial vertical angle (CVA), B: cranial rotation angle (CRA)

to place his/her head in the most comfortable position. The subject was in a comfortable standing position with the cervical, shoulder, and upper extremity muscles relaxed. The lateral view was photographed using a DSC-H50 (SONY, Japan) digital camera. The distance between the camera and the measurer was set at $1.5 \mathrm{~m}$. CVA was measured on images captured of patients in SBP by marking C7, the tragus of the ear, and the lateral canthus of the eye, and then drawing a horizontal line passing through $\mathrm{C} 7$ making a right angle with the vertical. Then, the angle between the line connecting $\mathrm{C} 7$ with the tragus of the ear and the horizontal line, CVA, was measured. CRA was determined by measuring the angle formed by the line connecting $\mathrm{C} 7$ with the tragus of the ear and the line connecting the tragus of the ear with the lateral canthus of the eye ${ }^{8)}$.

The experimental results were statistically analyzed using SPSS 15.0 KO (SPSS, Chicago, IL, USA). After the general characteristics of the subjects were determined, the paired t-test was used to compare the changes in the CVA and CRA between pre- and post-intervention within each group. The significance of the difference between the two groups was investigated with the independent $t$-test. The statistical significance level, $\alpha$, was chosen as 0.05 .

\section{RESULTS}

In the experimental group, CVA significantly increased $(p<0.05)$, while CRA significantly decreased $(p<0.05)$. Similarly, in the control group, CVA significantly increased $(p<0.05)$, while CRA significantly decreased $(p<0.05)$. In the comparison between the two groups, the experimental group showed a more significant increase in CVA $(p<0.05)$ and a more significant decrease in CRA $(p<0.05)$ than the control group (Table 1).

\section{DISCUSSION}

In the present study, cervical mobilization combined with thoracic mobilization was performed for patients with FHP, and changes in FHP were compared. 
Table 1. Comparison of CVA and CRA in the experimental and control groups

\begin{tabular}{ccccc}
\hline \multirow{2}{*}{ Variables } & \multicolumn{2}{c}{ Experimental group $(\mathrm{n}=15)$} & \multicolumn{2}{c}{ Control group $(\mathrm{n}=15)$} \\
\cline { 2 - 5 } & Before & After & \multicolumn{1}{c}{ Before } & \multicolumn{1}{c}{ After } \\
\hline $\mathrm{CVA}^{\dagger}$ & $46.6 \pm 3.3$ & $48.9 \pm 3.1^{*}$ & $45.8 \pm 2.5$ & $46.7 \pm 2.4^{*}$ \\
$\mathrm{CRA}^{\dagger}$ & $155.3 \pm 3.1$ & $152.6 \pm 3.1^{*}$ & $155.6 \pm 3.2$ & $154.5 \pm 3.1^{*}$ \\
\hline
\end{tabular}

Mean $\pm \mathrm{SD}, * \mathrm{p}<0.05$ : paired t-test, $\dagger \mathrm{p}<0.05$ : ANCOVA test, CVA: cranial vertical angle, CRA: cranial rotation angle

Gong et al. ${ }^{4}$ reported that cervical mobilization performed for 40 students with abnormal posture of the cervical spine significantly improved FHP. Bronfort et al. ${ }^{10)}$ also reported that spinal manual therapy combined with exercise, used to treat 191 chronic neck pain patients, resulted in significantly increased ROM. In another study, in which traction combined with cervical manipulation was used to treat patients with reduced cervical lordosis and severe FHP, it was reported that cervical lordosis increased while FHP decreased ${ }^{11)}$. It is generally considered that these improvements were derived from the effect of spinal manual therapy that extended tissues around the joints and increased the apophyseal joints' mobility, thereby recovering cervical lordosis as well as affecting posterior movements of the head. In another study, cervical functions were significantly improved when thrust mobilization/manipulation was performed on the thoracic spines of 60 patients with neck pain $^{12)}$. Similarly, Krauss et al. ${ }^{13)}$ reported that upper thoracic translatoric spinal manipulation, performed for 22 patients, relieved cervical pain and improved patients' ROM. Given these results, the movements of the thoracic spine appear to affect pain in the cervical spine as well as ROM and posture of the cervical spine. In the present study, CVA increased and CRA decreased in both the group receiving only cervical mobilization and the group receiving both cervical and thoracic mobilization. Thus, FHP improved in both groups. However, when both groups were compared, the results indicate that FHP was more significantly improved in the group receiving both cervical and thoracic mobilization.

Given these results, it can be said that although cervical mobilization and cervical mobilization combined with thoracic mobilization are both effective for FHP recovery, the combination of cervical and thoracic mobilization is more effective. Since FHP not only involves cervical spine deformation, but is also accompanied by upper thoracic spine deformation, more effective recovery of cervical spine alignment can be expected from treatment that includes the upper thoracic spine.

Therefore, we recommend cervical mobilization combined with thoracic mobilization as a clinical intervention for neck pain patients with FHP rather than cervical mobilization alone, and related studies should be conducted with larger numbers of subjects.

\section{REFERENCES}

1) Kraus SL: TMJ Disorders: Management of the Craniomandibular Complex. New York: Churchill Livingstone Press, 1994, pp 325-412.

2) Jung NS, Choi KH: Isometric evaluation of the flexors, extensors and lateral flexors of the cervical. The Journal of Korean Academy of Physical Therapist, 1994, 1: 3-10.

3) Mulligan BR: Manual Therapy: "NAGS", "SNAGS", "MWMS" etc. New Zealand: Plane View Press, 2006, pp 45-55.

4) Gong WT, Hwang BG, Lee YM: The effects of Gong's mobilization on cervical lordosis, forward head posture and cervical ROM in abnormal posture of cervical spine of college students. J Phys Ther Sci, 2011, 23: 531-534. [CrossRef]

5) Jung YW: Effects of McKenzie exercise on the functional recovery and forward head posture of chronic neck pain patients. Korean Soc Phys Med, 2006, 1: 93-108.

6) Saring-Bahat H: Evidence for exercise therapy in mechanical neck disorders. 2003, 8: 10-20.

7) Terrett AC, Vernon H: Manipulation and pain tolerance: a controlled study of the effect of spinal manipulation on paraspinal cutaneous pain tolerance levels. Am J Phys Med, 1984, 63: 217-225. [Medline]

8) Lee KS, Jung HY: Analysis of the change of the forward head posture according to computer using time. Korean Soc Phys Med, 2009, 4: 117-124.

9) Kim GC, Lee SY, Jeong HJ: Effects of upper thoracic joint mobilization on dynamic stability of patients with chronic neck pain. J Phys Ther Sci, 2011, 23: 753-756. [CrossRef]

10) Bronfort G, Evans R, Nelson B, et al.: A randomized clinical trial of exercise and spinal manipulation for patients with chronic neck pain. Spine, 2001, 26: 788-797. [Medline] [CrossRef]

11) Harrison DE, Harrison DD, Betz JJ, et al.: Increasing the cervical lordosis with chiropractic biophysics seated combined extension-compression and transverse load cervical traction with cervical manipulation: nonrandomized clinical control trial. J Manipulative Physiol Ther, 2003, 26: 139-151. [Medline] [CrossRef]

12) Cleland JA, Glynn P, Whitman JM, et al.: Short-term thrust versus nonthrust mobilization/manipulation directed at the thoracic spine in patients with neck pain: a randomized clinical trial. Phys Ther, 2007, 87: 431-440. [Medline] [CrossRef]

13) Krauss J, Creighton D, Ely JD, et al.: The immediate effects of upper thoracic translatoric spinal manipulation on cervical pain and range of motion: a randomized clinical trial. J Man Manip Ther, 2008, 16: 93-99. [Medline] [CrossRef] 